\title{
LINEARIZATION OF THE RIEMANN PROBLEM FOR A TRIANGULAR SYSTEM OF CONSERVATION LAWS AND DELTA SHOCK WAVE FORMATION PROCESS
}

\author{
V. BOJKOVIC, V.G. DANILOV, AND D. MITROVIC
}

\begin{abstract}
We generalize procedure from [28] on the case of more general triangular system of conservation laws arising from so called generalized pressureless gas dynamics. Using the weak asymptotic method [10, 12], more precisely nonlinear superposition law [8], we approximate the nonlinear system by a linear one. Then, we can use usual method of characteristics to find approximate solution to the original system. As a consequence, we shall see how delta shock wave naturally arises along the characteristics.
\end{abstract}

\section{INTRODUCTION}

In recent years, the weak asymptotic method has been applied on many problems involving formation and interaction of nonlinear waves. For instance, using the method we are able to find explicit formulas describing: interaction of solitons in the case of generalized $\mathrm{KdV}$ equation [9], interaction [10] and formation [7] of $\delta$-shock wave in the case of triangular system of conservation laws, confluence of free boundaries in the Stefan problem with underheating [5]... Here, we want to cast another, we believe, interesting and important light on the possibilities of the method.

The subject of the current paper is the following triangular system of conservation laws:

$$
\begin{aligned}
& u_{t}+(f(u))_{x}=0, \\
& v_{t}+(v g(u))_{x}=0,
\end{aligned}
$$

augmented with the Riemann initial data:

$$
\begin{aligned}
& \left.u\right|_{t=0}= \begin{cases}U_{L}, & x<0 \\
U_{R}, & x \geq 0\end{cases} \\
& \left.v\right|_{t=0}= \begin{cases}V_{L}, & x<0 \\
V_{R}, & x \geq 0\end{cases}
\end{aligned}
$$

where $U_{R}<U_{L}$.

This Riemann problem is intensively investigated in recent years $[7,10,11,13,14$, $16,17,18,19,21,24,28,30,31,32,36,37,39]$ (the list is far from being complete). The reason for this lies in applicability of the system - it arises from (generalized)

1991 Mathematics Subject Classification. 35L65, 35L67.

Key words and phrases. linearization, delta shock wave formation; non-strictly hyperbolic system; global approximate solution; weak asymptotic method.

The work of V.D. is supported by RFFI grant 05-01-00912, DFG Project 436 RUS 113/895/0-1.

The work of D.M. is supported in part by the local government of the municipality Budva. 
pressureless gas dynamics [3, 27]. Another, purely mathematical reason, is the fact that under the following assumptions on $f$ and $g$ (see e.g. [7] for derivation of the conditions):

$$
\begin{aligned}
& f \in C^{2}\left(\left[U_{R}, U_{L}\right]\right), \quad g \in C^{1}\left(\left[U_{R}, U_{L}\right]\right), \\
& f^{\prime \prime}>0 \quad \text { on }\left[U_{R}, U_{L}\right], \\
& g^{\prime}-f^{\prime \prime} \geq 0 \quad \text { on }\left[U_{R}, U_{L}\right], \\
& \exists \hat{U} \in\left(U_{R}, U_{L}\right) \text { such that } g(\hat{U})=f^{\prime}(\hat{U})
\end{aligned}
$$

it does not admit classical BV solution. Clearly, this raises challenging mathematical questions.

Informally speaking, we have Dirac $\delta$ distribution as a component of solution to the considered problem. In order to formalize this situation, several concepts allowing Dirac $\delta$ distribution as a solution to the problem are introduced. We gave detailed description of different concepts in [7]. Also, one can found lots of information on this issue in [10]. Here, we will just mention possible approaches in formalizing existence of very singular objects (such as Dirac distribution) as a solution or even as a coefficient of an equation.

One can define solution to the problem by extending definition of Radon measures (they are defined over the set of continuous functions) on the set of $B V$ functions as the set of test functions (see e.g. [13, 16, 17, 18, 19, 36, 39]).

Also, one can consider $\delta$ distribution as a family of smooth functions weakly converging to $\delta$, and work with the family as with ordinary smooth functions $[22$, 31].

Finally, in $[10,12]$ authors gave definition of the solution to a Cauchy problem for system (1), (2) which allows mentioned singular objects as solutions to the problem and which rather naturally generalizes classical definition of the weak solution.

In the rest of the introduction we will focus our attention on a method of linearization that we are proposing here.

The notion of linearization can be understood in two senses.

The first one consists in finding linear properties of nonlinear operations. Such principle is applied in the case of famous compensated compactness [29, 38]. Corner stone of this method is lemma stating about necessary conditions under which two sequences $\left(u_{n}\right)_{n},\left(v_{n}\right)_{n} \subset L^{2}(\Omega), \Omega \subset \mathbb{R}^{d}$, converging weakly in $L^{2}(\Omega)$ to $u, v \in$ $L^{2}(\Omega)$ satisfy

$$
u_{n} v_{n} \rightarrow u v \text { in } \mathcal{D}^{\prime}(\Omega) .
$$

Similar logic, i.e. finding linear properties of nonlinear objects, is used in deriving the nonlinear superposition law (see Theorem 3).

The other meaning of linearization, which is interesting to us at the moment, is reducing a problem of solving nonlinear equation to a problem of solving linear equation.

For instance, in the case of Dirichlet problem for quasilinear elliptic equation (see e.g. [15]), one first finds apriori inequalities for solutions of appropriate linear equations and then uses fixed point theorems to conclude about existence of the solution to the original nonlinear problem. So, the nonlinearity which appears in the equation is replaced by the linear equation and a problem of finding fixed point.

Another example, which is more corresponding to our situation, is reducing scalar conservation law to a transport equation. In the famous kinetic approach 
[34], using admissibility conditions from [23], nonlinearity is replaced by a "bad" righthand side. More precisely, it can be shown that an entropy admissible solution to the following scalar conservation law

$$
\partial_{t} u+\partial_{x} f(u)=0
$$

satisfies at the same time the following linear equation

$$
\partial_{t} h(t, x, \xi)+\partial_{x} f^{\prime}(\xi) h(t, x, \xi)=-\partial_{\xi} m(t, x, \xi),
$$

where $m$ is a positive measure and

$$
h(t, x, \xi)= \begin{cases}1, & \xi \leq u(x, t) \leq 0 \\ -1, & 0 \leq u(x, t) \leq \xi \\ 0, & \text { else. }\end{cases}
$$

So, we see that we have lost nonlinearity but we have problematic term on the righthand side of the new equation. Still, it appears that it is much easier end efficient to operate with such linear equation then the nonlinear one (see $[2,20,35])$.

Similar approach exists in the case of hyperbolic systems and it enables achieving substantially new results $[25,26,33]$. But, as in the case of scalar conservation law, in order to linearize the system we need existence of infinitely many entropies corresponding to the system.

Here, we propose method of linearization which is independent on existence of entropies but it can be applied only in the case of special initial data. Similarly as in the kinetic approach, we "replace" nonlinearity by a "bad" righthand side (compare (2), (4) and (26), (27)). But, unlike the situation that we have in the kinetic approach, first, Cauchy problems (1)-(4) and (18),(26), (19),(27) are not completely equivalent, and second, Cauchy problem $(18),(26),(19),(27)$ is not linear. More precisely, a solution to problem $(18),(26),(19),(27)$ is only approximate solution to the original Riemann problem (1)-(4). Concerning nonlinearity of Cauchy problem $(18),(26),(19),(27)$, as we will see, it disappears after negligible time (for $t=\mathcal{O}\left(\varepsilon^{1 / 2}\right)$ ) and it serves to us only to determine the right hand side in equations corresponding to (18),(26), (19),(27). Thus, we can say that Cauchy problem $(18),(26),(19),(27)$ is "almost linear".

Also notice that in [7] we reduced system (1),(2) with a continuous initial data on an "almost linear" Cauchy problem, but the linearization was due to the fact that the system is linear in $v$. Here, we propose a method which is independent on the linearity with respect to $v$.

Finally, notice that this method will give in the limit an admissible weak solution to the considered problem (see $[7,8]$ ).

We shall briefly describe the method of linearization which we shall use.

First, we shall consider equation (1).

In general, classical solutions to hyperbolic conservation laws can be obtained by the method of characteristics. Such solutions exist until characteristics do not intersect each other. The moment of the first intersection of characteristics is usually called the gradient catastrophe and in that moment singularity of the solution is formed. Also, in that moment we have to pass to the concept of weak solution.

But, if we would somehow succeed to avoid intersection of characteristics, and then write down the equation to which those new characteristics correspond, then the new equation should be linear. In order to explain this idea more precisely, 
consider equation (1) with the following perturbed Riemann initial data:

$$
\left.u\right|_{t=0}=\hat{u}(x)= \begin{cases}U_{L}, & x<a_{2} \\ u_{0}(x), & a_{2} \leq x \leq a_{1} \\ U_{R}, & a_{1}<x\end{cases}
$$

where $a_{1}=\varepsilon^{1 / 2}$ and $a_{2}=-\varepsilon^{1 / 2}$ for a small parameter $\varepsilon$, while $u_{0}$ is such that

$$
f^{\prime}\left(u_{0}(x)\right)=-K_{1} x+b_{1}
$$

for a constants $K_{1}>0$ and $b_{1}$.

Characteristics of Cauchy problem (1), (6) are plotted on Figure 1. Notice that all the characteristics emanating from $\left(-\varepsilon^{1 / 2}, \varepsilon^{1 / 2}\right)$ intersect in the same point. In order to avoid their intersection, natural idea is to smear the discontinuity line, i.e. to take $\varepsilon$ neighborhood of the discontinuity line and to dispose characteristics in that neighborhood in a way that they do not intersect each other, and as $\varepsilon \rightarrow 0$ all of them lump together into the discontinuity line. Of course, this will not be the standard characteristics for problem (1), (3). Nevertheless, along them approximate solution to our problem will remain constant. Such lines we call 'new characteristics' (see Figure 2 and $[7,8]$ ). Also notice that we replaced nonlinear equation (1) by a family of linear equations indexed by the small parameter $\varepsilon \rightarrow 0$ (they are nonlinear only for a short period of time).

This idea is formalized in [7]. Therefore, in Section 3 we shall just briefly describe the method that we used in [7] in order to linearize and solve (1). At the end of the section we formulate the main theorem.

In Section 2 we give auxiliary notions and notations.

In Section 4 we consider equation (2). We use completely the same procedure as in Section 3; we replace nonlinear equation (2) by a family of (almost) linear equations (26) admitting the family of solutions $\left(v_{\varepsilon}\right)_{\varepsilon}$. But, unlike equation (1), we linearize (2) with expense to "bad" righthand side (more precisely, the righthand side will be regularized $\delta$ distribution). As a consequence, we will obtain explicit formulas representing global approximate solution to problem (2), (4), and we will see how $\delta$-shock wave naturally arises along 'new characteristics'. More precisely, we will prove that $\left(v_{\varepsilon}\right)_{\varepsilon}$ converges to a distribution containing $\delta$ distribution. This is in accordance with the previous result on this subject (see $[10,14,16,17,31]$ and many others).

In Section 5 we give directions concerning possible usage of the method presented here on an arbitrary system of conservation laws.

\section{Auxiliary notions and statements}

To solve our problem we will use the weak asymptotic method $[9,10]$. Therefore, we provide basic notions and statements of the method.

Definition 1. [10] By $O_{\mathcal{D}^{\prime}}\left(\varepsilon^{\alpha}\right) \in \mathcal{D}^{\prime}(\mathbb{R}), \alpha \in \mathbb{R}$, we denote the family of distributions depending on $\varepsilon \in(0,1)$ and $t \in \mathbb{R}^{+}$such that for any test function $\eta(x) \in C_{0}^{1}(\mathbb{R})$, the estimate

$$
\left\langle O_{\mathcal{D}^{\prime}}\left(\varepsilon^{\alpha}\right), \eta(x)\right\rangle=O\left(\varepsilon^{\alpha}\right), \quad \varepsilon \rightarrow 0,
$$

holds, where the estimate on the right-hand side is understood in the usual sense and locally uniformly in $t$, i.e., $\left|O\left(\varepsilon^{\alpha}\right)\right| \leq C_{T} \varepsilon^{\alpha}$ for $t \in[0, T]$. 


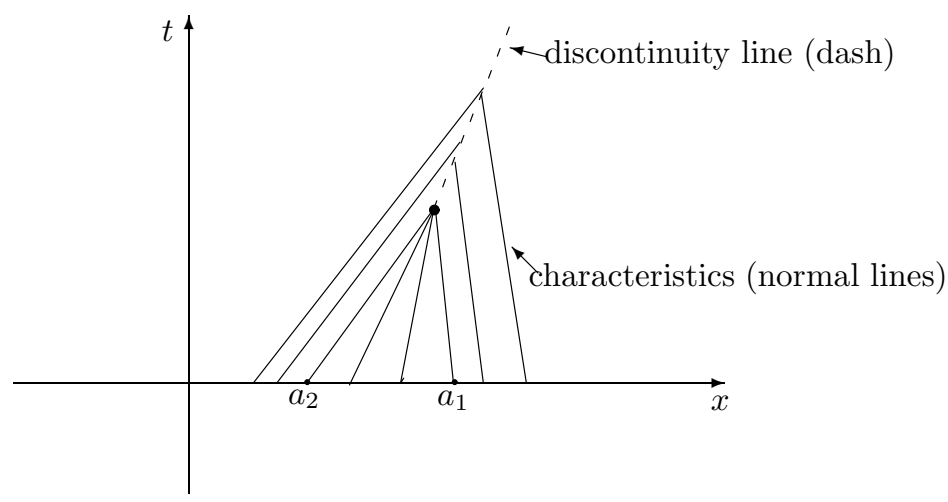

FiguRE 1. Standard characteristics for (1), (19). Dotted point in $(t, x)$ plane is $\left(t^{*}, x^{*}\right)$.

Now, we can give definition of our approximating solution:

Definition 2. [7] The family of pairs of functions $\left(u_{\varepsilon}, v_{\varepsilon}\right)=\left(u_{\varepsilon}(x, t), v_{\varepsilon}(x, t)\right)$, $\varepsilon>0$, is called a weak asymptotic solution of problem (1-4) if for an $\alpha>0$ we have

$$
\begin{gathered}
u_{\varepsilon t}+\left(f\left(u_{\varepsilon}\right)\right)_{x}=\mathcal{O}_{\mathcal{D}^{\prime}}\left(\varepsilon^{\alpha}\right), \\
v_{\varepsilon t}+\left(v_{\varepsilon} g\left(u_{\varepsilon}\right)\right)_{x}=\mathcal{O}_{\mathcal{D}^{\prime}}\left(\varepsilon^{\alpha}\right), \\
\left.u_{\varepsilon}\right|_{t=0}-\left.u\right|_{t=0}=\mathcal{O}_{\mathcal{D}^{\prime}}\left(\varepsilon^{\alpha}\right),\left.\quad v_{\varepsilon}\right|_{t=0}-\left.v\right|_{t=0}=\mathcal{O}_{\mathcal{D}^{\prime}}\left(\varepsilon^{\alpha}\right), \quad \varepsilon \rightarrow 0 .
\end{gathered}
$$

The following theorem is basic in our construction. It is called nonlinear superposition law. Actually, it states about linear properties of the operation of superposition of nonlinear functions.

Theorem 3. [7] Let $\omega_{i} \in C_{0}^{\infty}(\mathbb{R}), i=1,2$, where $\lim _{z \rightarrow+\infty} \omega_{i}(z)=1$,

$\lim _{z \rightarrow-\infty} \omega_{i}(z)=0$ and $\frac{d \omega(z)}{d z} \in \mathcal{S}(\mathbb{R})$ where $\mathcal{S}(\mathbb{R})$ is the Schwartz space of rapidly decreasing functions. For a bounded functions $a, b, c$ defined on $\mathbb{R}^{+} \times \mathbb{R}$ and bounded functions $\varphi_{i}, i=1,2$, defined on $\mathbb{R}^{+}$, we have

$$
\begin{aligned}
& f\left(a+b \omega_{1}\left(\frac{\varphi_{1}-x}{\varepsilon}\right)+c \omega_{2}\left(\frac{\varphi_{2}-x}{\varepsilon}\right)\right) \\
= & f(a)+H\left(\varphi_{1}-x\right)\left(f(a+b+c) B_{1}+f(a+b) B_{2}-f(a+c) B_{1}-f(a) B_{2}\right) \\
& +H\left(\varphi_{2}-x\right)\left(f(a+b+c) B_{2}-f(a+b) B_{2}+f(a+c) B_{1}-f(a) B_{1}\right)+\mathcal{O}_{\mathcal{D}^{\prime}}(\varepsilon),
\end{aligned}
$$

where $H$ is the Heaviside function and $B_{i}=B_{i}\left(\frac{\varphi_{2}-\varphi_{1}}{\varepsilon}\right)$ is such that for every $\rho \in \mathbb{R}$ we have

$$
B_{1}(\rho)=\int \dot{\omega}_{1}(z) \omega_{2}(z+\rho) d z \text { and } B_{2}(\rho)=\int \dot{\omega}_{2}(z) \omega_{1}(z-\rho) d z
$$

and

$$
B_{1}(\rho)+B_{2}(\rho)=1 .
$$


Furthermore, we have:

$$
\begin{aligned}
& B_{1}(\rho)=1-B_{2}(\rho) \rightarrow 1, \text { as } \rho \rightarrow+\infty \\
& B_{1}(\rho)=1-B_{2}(\rho) \rightarrow 0, \text { as } \rho \rightarrow-\infty
\end{aligned}
$$

\section{Linearization of Riemann problem (1), (3)}

In this section we explain how to find approximate solution to problem (1), (3) by linearizing the equation. The theorem is formulated at the end of the section and we leave it without proof. The proof can be found in [7].

In order to accomplish the linearization properly, on the first step we perturb initial data as given in (6). So, problem (1),(3) is initially replaced by family of problems (1), (6).

We introduce the notation that we shall use (as usual $x \in \mathbb{R}, t \in \mathbb{R}^{+}$):

$$
\begin{aligned}
B_{i} & =B_{i}(\rho), \quad \rho=\frac{\varphi_{2}-\varphi_{1}}{\varepsilon}, \quad \varphi_{i}=\varphi_{i}(t, \varepsilon), \\
H_{i} & =H\left(\varphi_{i}-x\right), \quad \delta_{i}=\delta\left(\varphi_{i}-x\right), \quad i=1,2, \\
\tau & =\frac{f^{\prime}\left(U_{L}\right) t+a_{2}-f^{\prime}\left(U_{R}\right) t-a_{1}}{\varepsilon}=\frac{\psi_{0}(t)}{\varepsilon}, \\
t^{*} & =\frac{a_{1}-a_{2}}{f^{\prime}\left(U_{L}\right)-f^{\prime}\left(U_{R}\right)}=\frac{2 \varepsilon^{1 / 2}}{f^{\prime}\left(U_{L}\right)-f^{\prime}\left(U_{R}\right)}, \\
x^{*} & =f^{\prime}\left(U_{L}\right) t^{*}+a_{2}=f^{\prime}\left(U_{R}\right) t^{*}+a_{1}=\frac{f^{\prime}\left(U_{R}\right) a_{1}-f^{\prime}\left(U_{R}\right) a_{2}}{f^{\prime}\left(U_{L}\right)-f^{\prime}\left(U_{R}\right)},
\end{aligned}
$$

where $H$ is the Heaviside function and $\delta$ Dirac distribution. We remind that $a_{2}=$ $-\varepsilon^{1 / 2}$ and $a_{1}=\varepsilon^{1 / 2}$.

The function $\tau$ is so-called 'fast variable'. It is equal to difference of standard characteristics of equation (1) emanating from $a_{2}$ and $a_{1}$, respectively. Since $a_{2}<$ $a_{1}$, when we are in the domain of existence of classical solution to (1), (6) we have $\tau \rightarrow-\infty$ as $\varepsilon \rightarrow 0$, while when we are in the domain where solution to (1), (6) is discontinuous (i.e. in the form of the shock wave) we have $\tau \rightarrow \infty$ as $\varepsilon \rightarrow 0$.

The point $\left(t^{*}, x^{*}\right)$ is the point of blow up of the classical solution to (1), (6).

The functions $\varphi_{i}, i=1,2$, are equations of the 'new characteristics' defined by (16) emanating from $a_{1}+\varepsilon A \frac{a_{1}+a_{2}}{2}$ and $a_{2}-\varepsilon A \frac{a_{1}+a_{2}}{2}$. They are given by the following globally solvable Cauchy problems (see [7]):

$$
\begin{aligned}
& \frac{d}{d t} \varphi_{1}(t, \varepsilon)=\left(B_{2}(\rho)-B_{1}(\rho)\right) f^{\prime}\left(U_{R}\right)+c B_{1}(\rho), \quad \varphi_{1}(0, \varepsilon)=a_{1}+A \varepsilon \frac{a_{1}-a_{2}}{2}, \\
& \frac{d}{d t} \varphi_{2}(t, \varepsilon)=\left(B_{2}(\rho)-B_{1}(\rho)\right) f^{\prime}\left(U_{L}\right)+c B_{1}(\rho), \quad \varphi_{2}(0, \varepsilon)=a_{2}-A \varepsilon \frac{a_{1}-a_{2}}{2},
\end{aligned}
$$

for large enough constant $A$.

It is well known that problem (1), (6) will have classical solution up to the moment $t^{*}$ given by:

$$
t=t^{*}=\max _{x \in\left(a_{2}, a_{1}\right)}-\frac{1}{f^{\prime \prime}\left(u_{0}(x)\right) u_{0}^{\prime}(x)}=\frac{1}{K_{1}},
$$

where $K_{1}$ is given by (7). The choice of our initial data is such that in the moment of blow up of the classical solution the shock wave will be formed and it will not change its shape for any $t>t^{*}$. This is because all the characteristics emanating from $\left[a_{2}, a_{1}\right]$ intersect in one point $\left(t^{*}, x^{*}\right)$ (see Figure 1 ). 
As we have already explained in the Introduction, in order to linearize equation (1), we need to perturb characteristics so that we avoid their intersection. More precisely, for every $\varepsilon>0$ we will dispose characteristics in an $\varepsilon$ neighborhood of the discontinuity line so that they do not intersect each other (see Figure 2). This perturbed characteristics we will call 'new characteristics'.

Then, we will write down the equation corresponding to such non-intersecting characteristics. Exactly this equation will be "almost" linear (i.e. nonlinearity will disappear after negligible time).

Another question that arises here is how to distribute 'new characteristics' in the $\varepsilon$ neighborhood of the discontinuity line. The obvious way to accomplish this is to distribute the 'new characteristics' uniformly in the mentioned area, i.e. in a way that every of them is parallel to the discontinuity line.

In [7] we used Theorem 3 and 'switch' functions $B_{i}, i=1,2$, appearing there to obtain the following equation of the 'new characteristics':

$$
\begin{aligned}
\dot{x} & =f^{\prime}\left(u_{\varepsilon}\right)\left(B_{2}(\rho)-B_{1}(\rho)\right)+c B_{1}(\rho), \quad \dot{u}_{\varepsilon}=0, \\
x(0) & =x_{0}+\varepsilon A\left(x_{0}-\frac{a_{1}+a_{2}}{2}\right), \quad u_{\varepsilon}(0)=\hat{u}\left(x_{0}\right), \quad x_{0} \in \mathbb{R} .
\end{aligned}
$$

The constant $A$ is such that for $x_{0} \in \mathbb{R}$ and every $t>0$ we have (equality below is easily deducible; concerning the inequality below, we address reader on [7]):

$$
\frac{\partial x}{\partial x_{0}}= \begin{cases}\frac{\varphi_{1}-\varphi_{2}}{a_{1}-a_{2}}, & x_{0} \in\left[a_{2}, a_{1}\right], \quad>0 . \\ 1, & \text { else }\end{cases}
$$

The Cauchy problem corresponding to characteristics (17) is

$$
\begin{aligned}
& \partial_{t} u_{\varepsilon}+\partial_{x}\left(\left(B_{2}-B_{1}\right) f\left(u_{\varepsilon}\right)+c B_{1} u_{\varepsilon}\right)=0 \\
& \left.u\right|_{t=0}=\hat{u}\left(x+\varepsilon\left(x-\frac{a_{1}+a_{2}}{2}\right)\right)=\hat{u}_{0}(x)+\mathcal{O}_{\mathcal{D}^{\prime}}(\varepsilon)
\end{aligned}
$$

for the function $\hat{u}$ given by (6).

Relation (17) means that 'new characteristics' do not mutually intersect which in turn means that there exists the solution $x_{0}=x_{0}(x, t, \varepsilon)$ of the implicit equation:

$$
x\left(x_{0}, t, \varepsilon\right)=x .
$$

Thus, the solution to Cauchy problem (18), (19) can be written in the form:

$$
u_{\varepsilon}(x, t)=\hat{u}\left(x_{0}(x, t, \varepsilon)\right) .
$$

Roughly speaking, the blow up of the gradient $\partial_{x} f\left(u_{\varepsilon}\right)$ will be neutralized by the term $B_{2}-B_{1}$. As we have seen in [7] (see also (23) below and proof of Corollary 5 ), the term $B_{2}-B_{1}$ will be close to zero after the gradient catastrophe thus eliminating influence of the nonlinearity $f\left(u_{\varepsilon}\right)$ appearing in (18).

We formalize the previous considerations in the following theorem which is proved in [7].

Theorem 4. Family of classical solution $u_{\varepsilon}, \varepsilon>0$, to the Cauchy problems (18), (19) is the weak asymptotic solution to Cauchy problem (1), (3) and it is given by

$$
u_{\varepsilon}(x, t)=\hat{u}\left(x_{0}(x, t, \varepsilon)\right),
$$




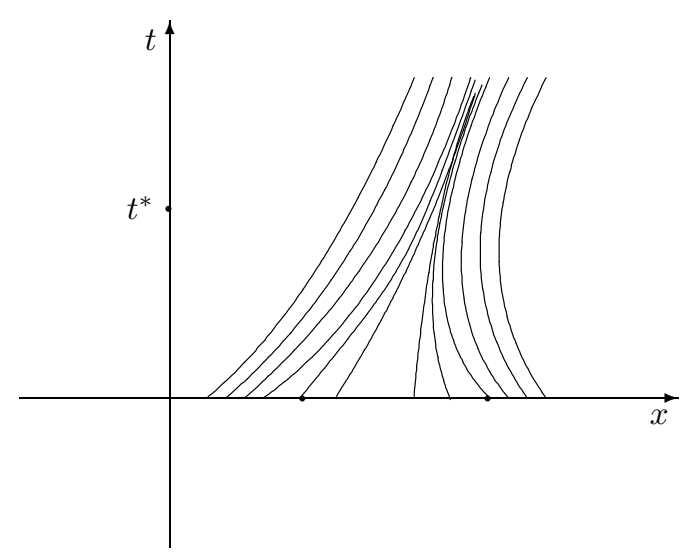

Figure 2. System of characteristics for $u_{\varepsilon}$ defined in Theorem 4 . The points $a_{1}+\varepsilon A \frac{a_{1}-a_{2}}{2}$ and $a_{2}-\varepsilon A \frac{a_{1}-a_{2}}{2}$ are dotted on the $x$ axis.

where $x_{0}$ is inverse function to the function $x=x\left(x_{0}, t, \varepsilon\right), t>0, \varepsilon>0$, of 'new characteristics' defined trough Cauchy problem (16). The functions $B_{1}$ and $B_{2}$ appearing in (16) are defined in Theorem 3, the constant $c$ is given by:

$$
c=2 \frac{f\left(U_{L}\right)-f\left(U_{R}\right)}{U_{L}-U_{R}},
$$

and $\rho=\rho\left(\psi_{0}(t) / \varepsilon\right)$ is the solution of the Cauchy problem:

$$
\rho_{\tau}=1-2 B_{1}(\rho),\left.\frac{\rho}{\tau}\right|_{\tau \rightarrow-\infty}=1 .
$$

Furthermore, it holds

$$
\begin{array}{r}
B_{1}(\rho(z)) \rightarrow 1 / 2, \quad z \rightarrow \infty \quad \text { and } \quad B_{1}(\rho(z)) \rightarrow 0, \quad z \rightarrow \infty \\
\left|\rho B_{1}(\rho)\right| \leq \text { const }<\infty, \quad \rho \in \mathbf{R}, \\
\left.\int_{0}^{\infty}\left(1-2 B_{1}(\rho(z))\right) d z<\infty, \quad \int_{-\infty}^{0} B_{1}(\rho(z))\right) d z<\infty .
\end{array}
$$

Corollary 5. For the weak asymptotic solution $\left(u_{\varepsilon}\right)$ of problem (1), (3) it holds for every fixed $t>0$ :

$$
u_{\varepsilon}(x, t) \rightarrow\left\{\begin{array}{ll}
U_{L}, & x<\frac{c t}{2}, \\
U_{R}, & x>\frac{c t}{2},
\end{array} \quad \text { in } \mathcal{D}^{\prime}(\mathbf{R}) \quad \text { as } \quad \varepsilon \rightarrow 0 .\right.
$$

Furthermore, we have for $i=1,2$ :

$$
\varphi_{i} \rightarrow \frac{c t}{2}, \quad \varepsilon \rightarrow 0
$$

Proof: From the classical ODE theory, one can see that for the global solution $\rho$ of problem (22)

$$
\rho \rightarrow \rho_{0} \quad \text { as } \tau \rightarrow+\infty
$$




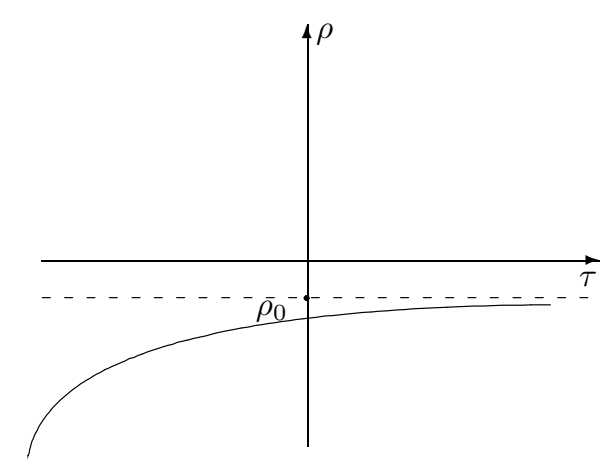

Figure 3. The curve represents solution of (22). Dot on the $\rho$ axis, denoted by $\rho_{0}$, is the smallest (and in this case unique) root of the equation $1-2 B_{1}(\rho)=0$.

where $\rho_{0}$ is stationary solution of the (22) (see Figure 3 ), i.e. constant such that $1-2 B_{1}\left(\rho_{0}\right)=0$, and therefore $B_{1}\left(\rho_{0}\right)=B_{2}\left(\rho_{0}\right)=\frac{1}{2}$ (we remind $\left.B_{1}+B_{2}=1\right)$.

Furthermore, notice that from $(5)$ we have $f^{\prime}\left(U_{L}\right)-f^{\prime}\left(U_{R}\right)>0$ and therefore for every fixed $t>0$ we have:

$$
\tau=\frac{\left(f^{\prime}\left(U_{L}\right)-f^{\prime}\left(U_{R}\right)\right) t-2 \varepsilon^{1 / 2}}{\varepsilon} \rightarrow \infty \text { as } \varepsilon \rightarrow 0 .
$$

From here and (25) it follows:

$$
\rho=\rho(\tau(t)) \rightarrow \rho_{0} \text { as } \varepsilon \rightarrow 0 .
$$

After letting $\varepsilon \rightarrow 0$ in (13) and (14), we obtain:

$$
\frac{d}{d t} \varphi_{1}(t, 0)=\frac{d}{d t} \varphi_{2}(t, 0)=\frac{c}{2}
$$

or, since $a_{i} \rightarrow 0, i=1,2$ :

$$
\varphi_{1}(t, 0)=\varphi_{2}(t, 0)=\frac{c}{2} t
$$

Since for $x<\varphi_{2}$ we have $u_{\varepsilon}(x, t)=U_{L}$, and for $x>\varphi_{1}$ we have $u_{\varepsilon}(x, t)=U_{R}$, we obtain in the limit:

$$
u_{\varepsilon}(x, t) \rightarrow\left\{\begin{array}{ll}
U_{L}, & x<\frac{c t}{2}, \\
U_{R}, & x>\frac{c t}{2},
\end{array} \quad \text { in } \mathcal{D}^{\prime}(\mathbf{R}) \text { as } \varepsilon \rightarrow 0\right.
$$

\section{Linearization of Riemann problem (1), (3)}

Following the method of Theorem 4, we will linearize problem (1), (3) by replacing it with the following family of problems: 


$$
\begin{aligned}
& \partial_{t} v_{\varepsilon}+\partial_{x}\left(g\left(u_{\varepsilon}\right)\left(B_{2}-B_{1}\right) v_{\varepsilon}+c B_{1} v_{\varepsilon}\right)_{x}=F(x, t, \varepsilon), \\
& \left.v_{\varepsilon}\right|_{t=0}=\hat{v}_{0}(x)= \begin{cases}V_{L}, & x<-\varepsilon^{1 / 2}-\varepsilon^{1 / 3} \\
v_{0}(x), & -\varepsilon^{1 / 2}-\varepsilon^{1 / 3} \leq x<\varepsilon^{1 / 2}+\varepsilon^{1 / 3} \\
V_{R}, & \varepsilon^{1 / 2}+\varepsilon^{1 / 3} \leq x,\end{cases}
\end{aligned}
$$

where $v_{0}$ is an arbitrary smooth function such that $\hat{v}_{0}$ is continuous function, and $F$ is chosen so that the arbitrary weak solution of (26) satisfies

$$
v_{\varepsilon t}+\left(g\left(u_{\varepsilon}\right) v_{\varepsilon}\right)_{x}=\mathcal{O}_{\mathcal{D}^{\prime}}\left(\varepsilon^{1 / 6}\right) .
$$

On the beginning, we will determine the function $F$. In order to do that, we first write system of characteristics for (26), (27):

$$
\begin{aligned}
& \dot{X}=g\left(u_{\varepsilon}\right)\left(B_{2}-B_{1}\right)+B_{1} c, \quad X(0)=x_{0} \in \mathbb{R} \\
& \dot{v}_{\varepsilon}=-g^{\prime}\left(u_{\varepsilon}\right)\left(B_{2}-B_{1}\right) v_{\varepsilon}+F(x, t, \varepsilon), \quad v_{\varepsilon}(0)=\hat{v}_{0}\left(x_{0}\right),
\end{aligned}
$$

where $u_{\varepsilon}=u_{\varepsilon}(X, t)$.

We focus our attention on (29). To show the global resoluteness of Cauchy problem $(26),(27)$, it is enough to prove that along the entire time axis we have

$$
\frac{\partial X}{\partial x_{0}}>0
$$

First, recall that from Theorem 4 we have:

$$
u_{\varepsilon}(x, t)=\hat{u}\left(\tilde{x}_{0}(x, t, \varepsilon)\right),
$$

where $\tilde{x}_{0}=\tilde{x}_{0}(x, t, \varepsilon)$ is inverse function to the function $x=x\left(x_{0}, t, \varepsilon\right)$ given by (16), and $\hat{u}$ is given by (6).

Having this in mind, after differentiating (29) in $x_{0}$ and using $B_{2}-B_{1}=1-2 B_{1}$ we get:

$$
\frac{d}{d t} \frac{\partial X}{\partial x_{0}}=\left(1-2 B_{1}\right) g^{\prime}\left(\hat{u}\left(\tilde{x}_{0}\right)\right) \hat{u}^{\prime}\left(\tilde{x}_{0}\right) \frac{\partial \tilde{x}_{0}}{\partial x} \frac{\partial X}{\partial x_{0}},
$$

where $B_{1}=B_{1}\left(\psi_{0}(t) / \varepsilon\right)$.

Then, integrating (31) with respect to the unknown function $\frac{\partial X}{\partial x_{0}}$ and having in mind that $\frac{\partial \tilde{x}_{0}}{\partial x}<\infty$ (see (17)), we obtain

$$
\frac{\partial X}{\partial x_{0}}=\exp \left(\int_{0}^{t} g^{\prime}(\hat{u}) \hat{u}^{\prime}\left(\tilde{x}_{0}\right)\left(B_{2}-B_{1}\right) \frac{\partial \tilde{x}_{0}}{\partial x} d t^{\prime}\right)>0
$$

Relation (32) implies global resoluteness of (26), (27), since it proves existence of the inverse function $x_{0}=x_{0}(X, t, \varepsilon)$ along entire temporal axis.

Consider now the characteristics given by (29) and emanating from $x_{0}=-\varepsilon^{1 / 2}-$ $\varepsilon^{1 / 3}$ and $x_{0}=\varepsilon^{1 / 2}+\varepsilon^{1 / 3}$, respectively:

$$
\begin{aligned}
& \dot{\varphi}_{2}^{*}=g\left(u_{\varepsilon}\right)\left(B_{2}-B_{1}\right)+B_{1} c \\
& \varphi_{2}^{*}(0, \varepsilon)=-\varepsilon^{1 / 2}-\varepsilon^{1 / 3},
\end{aligned}
$$

for $\varphi_{2}^{*}=\varphi_{2}^{*}(t, \varepsilon)$, and

$$
\begin{aligned}
& \dot{\varphi}_{1}^{*}=g\left(u_{\varepsilon}\right)\left(B_{2}-B_{1}\right)+B_{1} c \\
& \varphi_{1}^{*}(0, \varepsilon)=\varepsilon^{1 / 2}+\varepsilon^{1 / 3},
\end{aligned}
$$

for $\varphi_{1}^{*}=\varphi_{1}^{*}(t, \varepsilon)$. 
Next, we prove that for $\varepsilon$ small enough we have along entire temporal axis (see Figure 4):

$$
\varphi_{2}^{*}(t, \varepsilon) \leq \varphi_{2}(t, \varepsilon), \quad \varphi_{1}(t, \varepsilon) \leq \varphi_{1}^{*}(t, \varepsilon)
$$

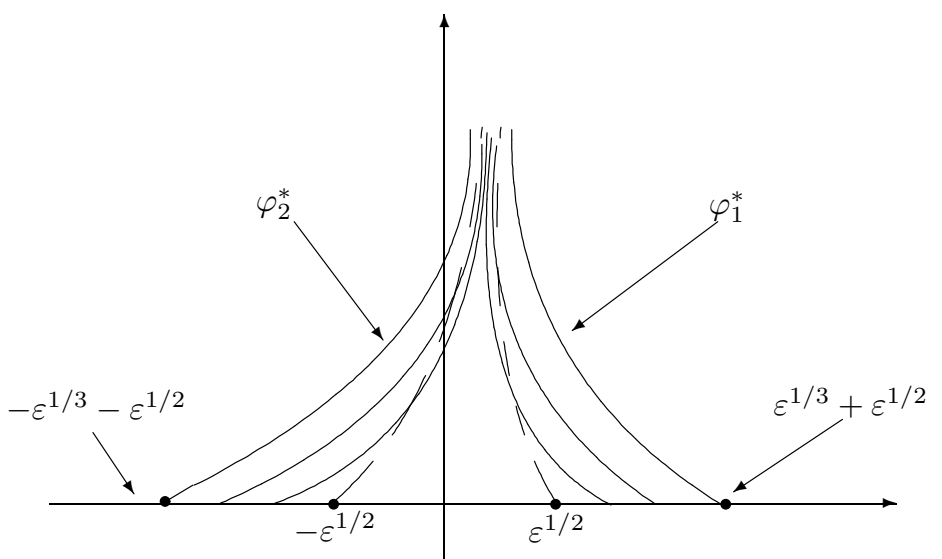

Figure 4. Dashed curves are the functions $\varphi_{i}, i=1,2$. Normal curves are characteristics defined by $(29)$. Note that they have the same slopes till the intersection with $\varphi_{i}, i=1,2$.

The solution of (33) is given by

$$
\varphi_{2}^{*}=\varphi_{2}^{*}(t, \varepsilon)=\int_{0}^{t}\left(g\left(u_{\varepsilon}\right)\left(B_{2}-B_{1}\right)+c B_{1}\right) d t^{\prime}-\varepsilon^{1 / 2}-\varepsilon^{1 / 3},
$$

while from (14) we see that, up to a (negligible) term of order $o(\varepsilon)$, the function $\varphi_{2}$ is given by :

$$
\varphi_{2}=\varphi_{2}(t, \varepsilon)=\int_{0}^{t}\left(f^{\prime}\left(U_{L}\right)\left(B_{2}-B_{1}\right)+c B_{1}\right) d t^{\prime}-\varepsilon^{1 / 2} .
$$

So, we have to prove that $\varphi_{2}-\varphi_{2}^{*}>0$, or, according to (36) and (37):

$$
\varphi_{2}-\varphi_{2}^{*}=\int_{0}^{t}\left(f^{\prime}\left(U_{L}\right)-g\left(u_{\varepsilon}\right)\right)\left(B_{2}-B_{1}\right) d t^{\prime}+\varepsilon^{1 / 3}>0 .
$$

Having in mind that $B_{2}-B_{1}=1-2 B_{1}$ and $B_{1}=B_{1}\left(\psi_{0}(t) / \varepsilon\right)$ the above expression can be transformed to

$$
\varphi_{2}-\varphi_{2}^{*}=\int_{0}^{t}\left(f^{\prime}\left(U_{L}\right)-g\left(u_{\varepsilon}\right)\right)\left(1-2 B_{1}\left(\rho\left(\frac{\psi_{0}(t)}{\varepsilon}\right)\right)\right) d t^{\prime}+\varepsilon^{1 / 3} .
$$

Next, having in mind the following change of variables:

$$
\begin{aligned}
& \frac{\psi_{0}\left(t^{\prime}\right)}{\varepsilon}=z \Longrightarrow\left(f^{\prime}\left(U_{L}\right)-f^{\prime}\left(U_{R}\right)\right) d t^{\prime}=\varepsilon d z \\
& 0<t^{\prime}<t \Longrightarrow 0<z<\frac{\psi_{0}\left(t^{\prime}\right)}{\varepsilon},
\end{aligned}
$$


we have

$$
\begin{aligned}
& \left|\int_{0}^{t}\left(1-2 B_{1}\left(\rho\left(\frac{\psi_{0}\left(t^{\prime}\right)}{\varepsilon}\right)\right)\right) d t^{\prime}\right|=\left|\left(\int_{0}^{t^{*}}+\int_{t^{*}}^{t}\right)\left(1-2 B_{1}\left(\rho\left(\frac{\psi_{0}\left(t^{\prime}\right)}{\varepsilon}\right)\right)\right) d t^{\prime}\right| \\
& =\mid \int_{0}^{t^{*}} d t^{\prime}-\varepsilon \frac{1}{f^{\prime}\left(U_{L}\right)-f^{\prime}\left(U_{R}\right)} \int_{\frac{\psi_{0}(0)}{\varepsilon}}^{0} B_{1}(\rho(z)) d z \\
& \quad+\varepsilon \frac{1}{f^{\prime}\left(U_{L}\right)-f^{\prime}\left(U_{R}\right)} \int_{0}^{\frac{\psi_{0}(t)}{\varepsilon}}\left(1-2 B_{1}(\rho(z))\right) d z \mid \\
& <\left|C_{3}\right| \varepsilon^{1 / 2}+\left|C_{4}\right| \varepsilon,
\end{aligned}
$$

where

$$
\begin{aligned}
& C_{3}=\frac{2}{f^{\prime}\left(U_{L}\right)-f^{\prime}\left(U_{R}\right)}<\infty \\
& C_{4}=-\frac{1}{f^{\prime}\left(U_{L}\right)-f^{\prime}\left(U_{R}\right)}\left(\int_{\psi_{0}(0) / \varepsilon}^{0} B_{1}(\rho(z)) d z-\int_{0}^{\psi_{0}(t) / \varepsilon}\left(1-2 B_{1}(\rho(z))\right) d z\right)<\infty,
\end{aligned}
$$

and this holds due to (23). From (38) and 40 we see that for $\varepsilon$ small enough we have fulfilled

$$
\varphi_{2}-\varphi_{2}^{*}=\varepsilon^{1 / 3}-\left|C_{3}\right| \varepsilon^{1 / 2}-\left|C_{4}\right| \varepsilon>0
$$

Similarly, we have $\varphi_{1}(t, \varepsilon)<\varphi_{1}^{*}(t, \varepsilon)$ for $\varepsilon$ small enough.

Notice that from the latter we have

$$
u_{\varepsilon}(x, t) \equiv \begin{cases}U_{L}, & x \leq \varphi_{2}^{*} \\ U_{R}, & x \geq \varphi_{1}^{*}\end{cases}
$$

Therefore, expressions for $\varphi_{i}^{*}, i=1,2$, become:

$$
\begin{aligned}
& \varphi_{1}^{*}=\int_{0}^{t}\left(g\left(U_{R}\right)\left(B_{2}-B_{1}\right)+c B_{1}\right) d t^{\prime}+\varepsilon^{1 / 2}+\varepsilon^{1 / 3} \\
& \varphi_{2}^{*}=\int_{0}^{t}\left(g\left(U_{L}\right)\left(B_{2}-B_{1}\right)+c B_{1}\right) d t^{\prime}-\varepsilon^{1 / 2}-\varepsilon^{1 / 3} .
\end{aligned}
$$

Now, it is easy to compute the distance between $\varphi_{i}^{*}, i=1,2$. We have:

$$
\varphi_{1}^{*}-\varphi_{2}^{*}=\int_{0}^{t}\left(g\left(U_{R}\right)-g\left(U_{L}\right)\right)\left(B_{2}-B_{1}\right) d t^{\prime}+2\left(\varepsilon^{1 / 2}+\varepsilon^{1 / 3}\right)=\mathcal{O}\left(\varepsilon^{1 / 3}\right),
$$

again relying on (40).

Similarly, we can compute $\varphi_{i}^{*}-\varphi_{i}, i=1,2$. We have from (4) and (42):

$$
\begin{aligned}
\varphi_{2}-\varphi_{2}^{*} & =\int_{0}^{t}\left(f^{\prime}\left(U_{L}\right)-g\left(U_{L}\right)\right)\left(B_{2}-B_{1}\right) d t^{\prime}+\varepsilon^{1 / 3}=\mathcal{O}\left(\varepsilon^{1 / 3}\right), \\
\varphi_{1}-\varphi_{1}^{*} & =\int_{0}^{t}\left(f^{\prime}\left(U_{R}\right)-g\left(U_{R}\right)\right)\left(B_{2}-B_{1}\right) d t^{\prime}-\varepsilon^{1 / 3}=\mathcal{O}\left(\varepsilon^{1 / 3}\right) .
\end{aligned}
$$

So, we have:

$$
\begin{aligned}
& \varphi_{2}-\varphi_{2}^{*}=\mathcal{O}\left(\varepsilon^{1 / 3}\right), \\
& \varphi_{1}-\varphi_{1}^{*}=\mathcal{O}\left(\varepsilon^{1 / 3}\right) .
\end{aligned}
$$


From here and (24) we also conclude:

$$
\varphi_{i}^{*}-\frac{c t}{2}=\mathcal{O}\left(\varepsilon^{1 / 3}\right) .
$$

Furthermore, if we assume that for every fixed $t$

$$
\operatorname{supp} F(x, t, \varepsilon) \subset\left(\varphi_{2}^{*}, \varphi_{1}^{*}\right)
$$

for every $t \in \mathbf{R}^{+}$from (42) and (26) we conclude:

$$
v_{\varepsilon}(x, t)=\left\{\begin{array}{ll}
V_{L}, & x<\varphi_{2}^{*}, \\
V_{R}, & x>\varphi_{1}^{*}
\end{array} .\right.
$$

Assumption (46) seems to be rather natural since out of the interval $\left(\varphi_{2}^{*}, \varphi_{1}^{*}\right)$ characteristics for $u_{\varepsilon}$ as well as for $v_{\varepsilon}$ behave rather nicely (they are parallel, and thus nonintersecting). Therefore, we do not have to "correct" anything out of $\left(\varphi_{2}^{*}, \varphi_{1}^{*}\right)$.

With the above assumption, we can determine the function $F(x, t, \varepsilon)$. So, assume that $\left(v_{\varepsilon}\right)_{\varepsilon}$ is the weak asymptotic solution to (26), (27) and that (46) holds. After adding and subtracting appropriate terms in (28), and using the fact that $B_{1}+B_{2}=$ 1, from (28) we have:

$$
\begin{aligned}
& v_{\varepsilon t}+\left(g\left(u_{\varepsilon}\right)\left(B_{2}-B_{1}\right) v_{\varepsilon}+c B_{1} v_{\varepsilon}\right)_{x} \\
& -F(x, t, \varepsilon)+\left(2 B_{1} g\left(u_{\varepsilon}\right) v_{\varepsilon}-c B_{1} v_{\varepsilon}\right)_{x}+F(x, t, \varepsilon)=\mathcal{O}_{\mathcal{D}^{\prime}}\left(\varepsilon^{1 / 6}\right) .
\end{aligned}
$$

From here and (26), it follows that

$$
B_{1}\left(c v_{\varepsilon}-2 g\left(u_{\varepsilon}\right) v_{\varepsilon}\right)_{x}=F(x, t, \varepsilon)+\mathcal{O}_{\mathcal{D}^{\prime}}\left(\varepsilon^{1 / 6}\right) .
$$

Now, we multiply (48) with $\eta(x) \in C_{0}^{1}(\mathbb{R})$, integrate over $\mathbb{R}$, and use partial integration to obtain

$$
B_{1} \int\left(2 g\left(u_{\varepsilon}\right) v_{\varepsilon}-c v_{\varepsilon}\right) \eta^{\prime}(x) d x=\int F \eta(x) d x+\mathcal{O}\left(\varepsilon^{1 / 6}\right)
$$

where $F=F(x, t, \varepsilon)$. Taking into account (35) and (47) we get from the last equality:

$$
\begin{aligned}
& B_{1}\left(\int_{-\infty}^{\varphi_{2}^{*}}\left(2 V_{L} g\left(U_{L}\right)-c V_{L}\right) \eta^{\prime}(x) d x\right. \\
& +\int_{\varphi_{2}^{*}}^{\varphi_{1}^{*}}\left(2 g\left(u_{\varepsilon}\right) v_{\varepsilon}-c v_{\varepsilon}\right) \eta^{\prime}(x) d x \\
& \left.+\int_{\varphi_{1}^{*}}^{+\infty}\left(2 V_{R} g\left(U_{R}\right)-c V_{R}\right) \eta^{\prime}(x) d x\right)=\int_{-\infty}^{+\infty} F \eta(x) d x+\mathcal{O}\left(\varepsilon^{1 / 6}\right)
\end{aligned}
$$

where $F=F(x, t, \varepsilon)$.

Later (see considerations below (60)), we shall prove that it is possible to choose the function $F$ so that

$$
\int_{\varphi_{2}^{*}}^{\varphi_{1}^{*}}\left(2 g\left(u_{\varepsilon}\right) v_{\varepsilon}-c v_{\varepsilon}\right) \eta^{\prime}(x) d x=\mathcal{O}\left(\varepsilon^{1 / 6}\right) .
$$


From here and since:

$$
\begin{aligned}
& B_{1} \int_{-\infty}^{\varphi_{2}^{*}}\left(2 V_{L} g\left(U_{L}\right)-c V_{L}\right) \eta^{\prime}(x) d x=B_{1}\left(2 V_{L} g\left(U_{L}\right)-c V_{L}\right) \eta\left(\varphi_{2}^{*}\right), \\
& B_{1} \int_{\varphi_{1}^{*}}^{+\infty}\left(2 V_{R} g\left(U_{R}\right)-c V_{R}\right) \eta^{\prime}(x) d x=-B_{1}\left(2 V_{R} g\left(U_{R}\right)-c V_{R}\right) \eta\left(\varphi_{1}^{*}\right),
\end{aligned}
$$

we conclude from (49):

$$
B_{1}\left[\left(2 V_{L} g\left(U_{L}\right)-c V_{L}\right) \eta\left(\varphi_{2}^{*}\right)-\left(2 V_{R} g\left(U_{R}\right)-c V_{R}\right) \eta\left(\varphi_{1}^{*}\right)\right]=\int F \eta(x) d x+\mathcal{O}\left(\varepsilon^{1 / 6}\right)
$$

We rewrite the last expression in the form:

$$
\begin{aligned}
& B_{1}\left[\left(c V_{R}-2 V_{R} g\left(U_{R}\right)+2 V_{L} g\left(U_{L}\right)-c V_{L}\right)\right] \eta\left(\varphi_{1}^{*}\right) \\
& +B_{1}\left(2 V_{L} g\left(U_{L}\right)-c V_{L}\right)\left(\eta\left(\varphi_{2}^{*}\right)-\eta\left(\varphi_{1}^{*}\right)\right)=\int F \eta(x) d x+\mathcal{O}\left(\varepsilon^{1 / 6}\right) .
\end{aligned}
$$

Then, recall that $B_{1}$ is bounded and notice that $\eta\left(\varphi_{2}^{*}\right)-\eta\left(\varphi_{1}^{*}\right)=\eta^{\prime}(\tilde{\varphi})\left(\varphi_{2}^{*}-\varphi_{1}^{*}\right)=$ $\mathcal{O}\left(\varepsilon^{1 / 6}\right)$, for appropriate $\tilde{\varphi} \in\left(\varphi_{2}^{*}, \varphi_{1}^{*}\right)$. So after denoting

$$
K=\left[\left(c V_{R}-2 V_{R} g\left(U_{R}\right)+2 V_{L} g\left(U_{L}\right)-c V_{L}\right)\right]
$$

we derive from (51) that $F$ should satisfy

$$
B_{1} K \eta\left(\varphi_{1}^{*}\right)=\int F \eta(x) d x+\mathcal{O}\left(\varepsilon^{1 / 6}\right)
$$

It is now clear that $F$ should represent regularization of Dirac $\delta$ function supported in $x=\varphi_{1}^{*}$.

Accordingly, denote $\tilde{\phi}=\frac{\varphi_{1}^{*}+\varphi_{2}^{*}}{2}$ and put

$$
F=F(x, t, \varepsilon)=B_{1} K \frac{1}{\varepsilon^{1 / 3}} \Phi\left(\frac{x-\tilde{\phi}}{\varepsilon^{1 / 3}}\right)
$$

where $\operatorname{supp} \Phi \subset(-1,1)$ and $\int \Phi(z) d z=1$. It is clear that the distributional limit of $(F(x, t, \varepsilon))_{\varepsilon}$ is $\delta$-distribution supported in $\lim _{\varepsilon \rightarrow 0} \varphi_{1}^{*}$. Now, we prove that such $F=F(x, t, \varepsilon)$ satisfies (53). Indeed, we have

$$
\begin{aligned}
B_{1} K \eta\left(\varphi_{1}\right) & =\int F \eta(x) d x+\mathcal{O}\left(\varepsilon^{1 / 6}\right) \\
& =B_{1} K \int \frac{1}{\varepsilon^{1 / 3}} \Phi\left(\frac{x-\tilde{\phi}}{\varepsilon^{1 / 3}}\right) \eta(x) d x+\mathcal{O}\left(\varepsilon^{1 / 6}\right) \\
& =\left(\frac{x-\tilde{\phi}}{\varepsilon^{1 / 3}}=z \Rightarrow d x=\varepsilon^{1 / 3} d z\right) \\
& =B_{1} K \int \Phi(z) \eta\left(\varepsilon^{1 / 3} z+\tilde{\phi}\right) d z+\mathcal{O}\left(\varepsilon^{1 / 6}\right) \\
& =B_{1} K \int \Phi(z)\left(\eta\left(\varphi_{1}^{*}\right)+\left(\varepsilon^{1 / 3} z+\frac{\varphi_{2}^{*}-\varphi_{1}^{*}}{2}\right) \eta^{\prime}(\tilde{z})\right) d z+\mathcal{O}\left(\varepsilon^{1 / 6}\right) \\
& =B_{1} K \eta\left(\varphi_{1}^{*}\right) \int \Phi(z) d z+B_{1} K \int \Phi(z)\left(\varepsilon^{1 / 3} z+\frac{\varphi_{2}^{*}-\varphi_{1}^{*}}{2}\right) \eta^{\prime}(\tilde{z}) d z+\mathcal{O}\left(\varepsilon^{1 / 6}\right) \\
& =B_{1} K \eta\left(\varphi_{1}^{*}\right)+\mathcal{O}\left(\varepsilon^{1 / 6}\right)
\end{aligned}
$$


where the last equality holds due to (43). So, (26) transforms into

$$
v_{\varepsilon t}+\left(g\left(u_{\varepsilon}\right)\left(B_{2}-B_{1}\right) v_{\varepsilon}+c B_{1} v_{\varepsilon}\right)_{x}=B_{1} K \frac{1}{\varepsilon^{1 / 3}} \Phi\left(\frac{x-\tilde{\phi}}{\varepsilon^{1 / 3}}\right) .
$$

To proceed, we compute $X^{-1}\left(\varphi_{i}\right), i=1,2$, where $X$ is equation of characteristics defined by (29). We have from the equation of characteristics (16) and (29), respectively, that $\varphi_{2}$ satisfies at the same time:

$$
\begin{aligned}
& \varphi_{2}=\int_{0}^{t}\left(\left(B_{2}-B_{1}\right) f^{\prime}\left(U_{L}\right)+c B_{1}\right) d t^{\prime}-\varepsilon^{1 / 2}, \\
& \varphi_{2}=\int_{0}^{t}\left(\left(B_{2}-B_{1}\right) g\left(U_{L}\right)+c B_{1}\right) d t^{\prime}+X^{-1}\left(\varphi_{2}\right) .
\end{aligned}
$$

Comparing the latter two expressions we get as before (see (40)):

$$
\begin{aligned}
X^{-1}\left(\varphi_{2}\right) & =\int_{0}^{t}\left(B_{2}-B_{1}\right)\left(f^{\prime}\left(U_{L}\right)-g\left(U_{L}\right)\right) d t^{\prime}-\varepsilon^{1 / 2} \\
& =\left(\frac{2\left(f^{\prime}\left(U_{L}\right)-g\left(U_{L}\right)\right)}{f^{\prime}\left(U_{L}\right)-f^{\prime}\left(U_{R}\right)}-1\right) \varepsilon^{1 / 2}+\mathcal{O}(\varepsilon) .
\end{aligned}
$$

Similarly,

$$
X^{-1}\left(\varphi_{1}\right)=\left(\frac{2\left(f^{\prime}\left(U_{R}\right)-g\left(U_{R}\right)\right)}{f^{\prime}\left(U_{L}\right)-f^{\prime}\left(U_{R}\right)}+1\right) \varepsilon^{1 / 2}+\mathcal{O}(\varepsilon)
$$

Now, we return to (29) and (30). We have from there:

$$
\begin{aligned}
X= & \int_{0}^{t}\left(g\left(u_{\varepsilon}\right)\left(B_{2}-B_{1}\right)+B_{1} c\right) d t^{\prime}+x_{0} \\
v_{\varepsilon}= & \exp \left(-\int_{0}^{t} g^{\prime}\left(u_{\varepsilon}\right) \frac{\partial u_{\varepsilon}}{\partial x}\left(B_{2}-B_{1}\right) d t^{\prime}\right)\left(\hat{v}\left(x_{0}\right)+\right. \\
& \left.+\int_{0}^{t} B_{1} K \frac{1}{\varepsilon^{1 / 3}} \Phi\left(\frac{X-\tilde{\phi}}{\varepsilon^{1 / 3}}\right) \exp \left(\int_{0}^{t} g^{\prime}\left(u_{\varepsilon}\right) \frac{\partial u_{\varepsilon}}{\partial x}\left(B_{2}-B_{1}\right)\right) d t^{\prime}\right),
\end{aligned}
$$

Recalling (32) we get from (57):

$$
\begin{aligned}
& X=\int_{0}^{t}\left(g\left(\hat{u}\left(\tilde{x}_{0}(X, t, \varepsilon)\right)\right)\left(B_{2}-B_{1}\right)+c B_{1}\right) d t^{\prime}+x_{0}, \\
& v_{\varepsilon}=\frac{1}{\frac{\partial X}{\partial x_{0}}}\left(\hat{v}\left(x_{0}\right)+\int_{0}^{t} B_{1} K \frac{1}{\varepsilon^{1 / 3}} \Phi\left(\frac{X-\tilde{\phi}}{\varepsilon^{1 / 3}}\right) \frac{\partial X}{\partial x_{0}}\left(x_{0}, t^{\prime}, \varepsilon\right) d t^{\prime}\right) .
\end{aligned}
$$

Since the new characteristics are parallel till the intersection with $\varphi_{i}(t, \varepsilon), i=$ 1,2 , we have for $t^{\prime} \leq t$

$$
X\left(x_{0}, t^{\prime}, \varepsilon\right)= \begin{cases}\int_{0}^{t^{\prime}}\left(g\left(U_{L}\right)\left(B_{2}-B_{1}\right)+c B_{1}\right) d t^{\prime \prime}+x_{0}, & x_{0}<X^{-1}\left(\varphi_{2}(t, \varepsilon)\right)<a_{2}, \\ \int_{0}^{t^{\prime}}\left(g\left(U_{R}\right)\left(B_{2}-B_{1}\right)+c B_{1}\right) d t^{\prime \prime}+x_{0}, & x_{0}>X^{-1}\left(\varphi_{1}(t, \varepsilon)\right)>a_{1},\end{cases}
$$

and thus

$$
\frac{\partial X}{\partial x_{0}}\left(x_{0}, t^{\prime}, \varepsilon\right)=1
$$

for $x_{0} \in\left(-\infty, X^{-1}\left(\varphi_{2}(t)\right)\right) \cup\left(X^{-1}\left(\varphi_{1}(t)\right), \infty\right)$ and $t^{\prime} \in[0, t)$. 
Using this, (41) and (47) we can rewrite $v_{\varepsilon}$ in the following form:

$$
v_{\varepsilon}(x, t)= \begin{cases}V_{L}, & x \leq \varphi_{2}^{*}, \\ V_{L}+\int_{0}^{t} B_{1} K \frac{1}{\varepsilon^{1 / 3}} \Phi\left(\frac{x-\tilde{\phi}}{\varepsilon^{1 / 3}}\right) d t^{\prime}, & \varphi_{2}^{*} \leq x<\varphi_{2}, \\ \frac{1}{\partial X}\left(\hat{v}\left(x_{0}\right)+\int_{0}^{t} B_{1} K \frac{1}{\varepsilon^{1 / 3}} \Phi\left(\frac{x-\tilde{\phi}}{\varepsilon^{1 / 3}}\right) \frac{\partial X}{\partial x_{0}}\left(x_{0}, t^{\prime}, \varepsilon\right) d t^{\prime}\right) & \\ \quad \text { for } x_{0}=-\int_{0}^{t}\left(g\left(u_{\varepsilon}\right)\left(B_{2}-B_{1}\right)+c B_{1}\right) d t^{\prime}+x, & \varphi_{2} \leq x<\varphi_{1}, \\ V_{R}+\int_{0}^{t} B_{1} K \frac{1}{\varepsilon^{1 / 3}} \Phi\left(\frac{x-\tilde{\phi}}{\varepsilon^{1 / 3}}\right) d t^{\prime}, & \varphi_{1} \leq x<\varphi_{1}^{*}, \\ V_{R}, & x \geq \varphi_{1}^{*} .\end{cases}
$$

Now, we can prove (50). We have:

$$
\begin{aligned}
& B_{1} \int_{\varphi_{2}^{*}}^{\varphi_{1}^{*}}\left(2 g\left(u_{\varepsilon}\right) v_{\varepsilon}-c v_{\varepsilon}\right) \eta^{\prime}(x) d x \\
& =B_{1} \int_{\varphi_{2}^{*}}^{\varphi_{2}}\left(2 g\left(U_{L}\right)-c\right) v_{\varepsilon} \eta^{\prime}(x) d x+B_{1} \int_{\varphi_{1}}^{\varphi_{1}^{*}}\left(2 g\left(U_{R}\right)-c\right) v_{\varepsilon} \eta^{\prime}(x) d x \\
& +B_{1} \int_{\varphi_{2}}^{\varphi_{1}}\left(2 g\left(u_{\varepsilon}\right)-c\right) v_{\varepsilon} \eta^{\prime}(x) d x \\
& =\left(x=X\left(x_{0}, t, \varepsilon\right) \Rightarrow d x=\frac{\partial X}{\partial x_{0}} d x_{0}\right) \\
& =B_{1} \int_{-\varepsilon^{1 / 2}-\varepsilon^{1 / 3}}^{X^{-1}\left(\varphi_{2}\right)}\left(2 g\left(U_{L}\right)-c\right) v_{\varepsilon}\left(x_{0}, t\right) \eta^{\prime}\left(X\left(x_{0}, t, \varepsilon\right)\right) d x_{0} \\
& +B_{1} \int_{X^{-1}\left(\varphi_{1}\right)}^{\varepsilon^{1 / 2}+\varepsilon^{1 / 3}}\left(2 g\left(U_{R}\right)-c\right) v_{\varepsilon}\left(x_{0}, t\right) \eta^{\prime}\left(X\left(x_{0}, t, \varepsilon\right)\right) d x_{0} \\
& +B_{1} \int_{X^{-1}\left(\varphi_{2}\right)}^{X^{-1}\left(\varphi_{1}\right)}\left(2 g\left(u_{\varepsilon}\right)-c\right) v_{\varepsilon}\left(x_{0}, t\right) \eta^{\prime}\left(X\left(x_{0}, t, \varepsilon\right)\right) \frac{\partial X}{\partial x_{0}}\left(x_{0}, t, \varepsilon\right) d x_{0},
\end{aligned}
$$

where we use (58) in the last equality. Consider now the integral

$$
\begin{aligned}
& B_{1} \int_{X^{-1}\left(\varphi_{2}\right)}^{X^{-1}\left(\varphi_{1}\right)}\left(2 g\left(u_{\varepsilon}\right)-c\right) v_{\varepsilon}\left(t, x_{0}\right) \eta^{\prime}\left(X\left(x_{0}, t, \varepsilon\right)\right) \frac{\partial X}{\partial x_{0}}\left(x_{0}, t, \varepsilon\right) d x_{0} \\
& =B_{1} \int_{X^{-1}\left(\varphi_{2}\right)}^{X^{-1}\left(\varphi_{1}\right)}\left(\hat{v}\left(x_{0}\right)+\int_{0}^{t} B_{1} K \frac{1}{\varepsilon^{1 / 3}} \Phi\left(\frac{X\left(x_{0}, t, \varepsilon\right)-\tilde{\phi}}{\varepsilon^{1 / 3}}\right) \frac{\partial X}{\partial x_{0}}\left(x_{0}, t^{\prime}, \varepsilon\right) d t^{\prime}\right) \eta^{\prime}(X) d x_{0} \\
& \leq C \frac{X^{-1}\left(\varphi_{1}\right)-X^{-1}\left(\varphi_{2}\right)}{\varepsilon^{1 / 3}}=\mathcal{O}\left(\varepsilon^{1 / 6}\right)
\end{aligned}
$$

where $C$ is constant independent on $\varepsilon$. 
Then, consider other two integrals in (60). First,

$$
\begin{aligned}
& B_{1} \int_{-\varepsilon^{1 / 2}-\varepsilon^{1 / 3}}^{X^{-1}\left(\varphi_{2}\right)}\left(2 g\left(U_{L}\right)-c\right) v_{\varepsilon}\left(x_{0}, t\right) \eta^{\prime}\left(X\left(x_{0}, t, \varepsilon\right)\right) d x_{0} \\
& =B_{1} \int_{-\varepsilon^{1 / 2}-\varepsilon^{1 / 3}}^{X^{-1}\left(\varphi_{2}\right)}\left(2 g\left(U_{L}\right)-c\right) \\
& \quad \times\left(V_{L}+\int_{0}^{t} \frac{1}{\varepsilon^{1 / 3}} \Phi\left(\frac{x_{0}+\int_{0}^{t^{\prime}}\left(g\left(U_{L}\right)-g\left(U_{R}\right)\right)\left(B_{2}-B_{1}\right) d t^{\prime \prime}}{\varepsilon^{1 / 3}}\right)\right. \\
& \left.\quad \times \frac{\partial X}{\partial x_{0}}\left(x_{0}, t, \varepsilon\right) d t^{\prime}\right) \eta^{\prime}\left(X\left(x_{0}, t, \varepsilon\right)\right) d x_{0} \\
& =B_{1} \int_{-\varepsilon^{1 / 2}-\varepsilon^{1 / 3}}^{X^{-1}\left(\varphi_{2}\right)}\left(2 g\left(U_{L}\right)-c\right) V_{L} \eta^{\prime}\left(X\left(x_{0}, t, \varepsilon\right)\right) d x_{0} \\
& +B_{1} \int_{-\varepsilon^{1 / 2}-\varepsilon^{1 / 3}}^{X^{-1}\left(\varphi_{2}\right)}\left(2 g\left(U_{L}\right)-c\right) \\
& \quad \times \int_{0}^{t} \frac{1}{\varepsilon^{1 / 3}} \Phi\left(\frac{x_{0}+\int_{0}^{t^{\prime}}\left(g\left(U_{L}\right)-g\left(U_{R}\right)\right)\left(B_{2}-B_{1}\right) d t^{\prime \prime}}{\varepsilon^{1 / 3}}\right) d t^{\prime} \eta^{\prime}\left(X\left(x_{0}, t, \varepsilon\right)\right) d x_{0} \\
& = \\
& \quad
\end{aligned}
$$

where the last two equalities hold due to (58). Then, denote:

$$
a(t, \varepsilon)=\int_{0}^{t}\left(g\left(U_{L}\right)-g\left(U_{R}\right)\right)\left(B_{2}-B_{1}\right) d t^{\prime}
$$

We introduce the following change of variables:

$$
\begin{aligned}
& \frac{x_{0}+a\left(t^{\prime}, \varepsilon\right)}{\varepsilon^{1 / 3}}=z \Rightarrow d x_{0}=\varepsilon^{1 / 3} d z \\
& x_{0} \in\left(-\varepsilon^{1 / 2}-\varepsilon^{1 / 3}, X^{-1}\left(\varphi_{2}\right)\right) \Rightarrow z \in\left(-\varepsilon^{1 / 6}-1, \frac{X^{-1}\left(\varphi_{2}\right)+a(t, \varepsilon)}{\varepsilon^{1 / 3}}\right),
\end{aligned}
$$

we get

$$
\begin{aligned}
& B_{1} \int_{-\varepsilon^{1 / 2}-\varepsilon^{1 / 3}}^{X^{-1}\left(\varphi_{2}\right)}\left(2 g\left(U_{L}\right)-c\right) v_{\varepsilon}\left(x_{0}, t\right) \eta^{\prime}\left(X\left(x_{0}, t, \varepsilon\right)\right) d x_{0} \\
& =B_{1}\left(2 g\left(U_{L}\right)-c\right) \int_{0}^{t} \int_{-\varepsilon^{1 / 6}-1}^{\frac{X^{-1}\left(\varphi_{2}\right)+a(t, \varepsilon)}{\varepsilon^{1 / 3}}} \Phi(z) \eta^{\prime}\left(X\left(x_{0}\left(z, t^{\prime}, \varepsilon\right), t, \varepsilon\right)\right) d z d t^{\prime}+\mathcal{O}\left(\varepsilon^{1 / 3}\right) \\
& =B_{1}\left(2 g\left(U_{L}\right)-c\right) \int_{0}^{t} \int_{-\varepsilon^{1 / 6}-1}^{\mathcal{O}\left(\varepsilon^{1 / 6}\right)} \Phi(z) \eta^{\prime}\left(X\left(x_{0}\left(z, t^{\prime}, \varepsilon,\right), t, \varepsilon\right)\right) d z d t^{\prime}+\mathcal{O}\left(\varepsilon^{1 / 3}\right),
\end{aligned}
$$


where the last equality holds due to (56). From (60), (62) and (63) we get

$$
\begin{aligned}
& B_{1} \int_{\varphi_{2}^{*}}^{\varphi_{2}}\left(2 g\left(U_{L}\right)-c\right) v_{\varepsilon} \eta^{\prime}(x) d x \\
& =B_{1} \int_{-\varepsilon^{1 / 2}-\varepsilon^{1 / 3}}^{X^{-1}\left(\varphi_{2}\right)}\left(2 g\left(U_{L}\right)-c\right) v_{\varepsilon}\left(x_{0}, t\right) \eta^{\prime}\left(X\left(x_{0}, t, \varepsilon\right)\right) d x_{0}+\mathcal{O}\left(\varepsilon^{1 / 3}\right) \\
& =t B_{1}\left(2 g\left(U_{L}\right)-c\right) \int_{-1}^{0} \Phi(z) d z \eta^{\prime}\left(\frac{c t}{2}\right)+\mathcal{O}\left(\varepsilon^{1 / 6}\right)
\end{aligned}
$$

since

$$
\eta^{\prime}\left(X\left(x_{0}, t, \varepsilon\right)\right)=\eta^{\prime}\left(\frac{c t}{2}\right)+\left(X\left(x_{0}, t, \varepsilon\right)-\frac{c t}{2}\right) \eta^{\prime \prime}(\tilde{X})=\eta^{\prime}\left(\frac{c t}{2}\right)+\mathcal{O}\left(\varepsilon^{1 / 3}\right),
$$

where $\tilde{X}$ is a point in the neighborhood of $\frac{c t}{2}$. Indeed, taking into account (45) and the fact that $x=X \in\left(\varphi_{2}^{*}, \varphi_{2}\right)$ we see that:

$$
\varphi_{2}^{*}<X\left(x_{0}, t, \varepsilon\right)<\varphi_{2} \Rightarrow X\left(x_{0}, t, \varepsilon\right)-\frac{c t}{2}=\mathcal{O}\left(\varepsilon^{1 / 3}\right),
$$

and this immediately gives (65). Similarly,

$$
\begin{aligned}
& B_{1} \int_{X^{-1}\left(\varphi_{1}\right)}^{\varepsilon^{1 / 2}+\varepsilon^{1 / 3}}\left(2 g\left(U_{R}\right)-c\right) v_{\varepsilon}\left(x_{0}, t\right) \eta^{\prime}\left(X\left(x_{0}, t, \varepsilon\right)\right) d x_{0} \\
& =B_{1}\left(2 g\left(U_{R}\right)-c\right) \int_{0}^{1} \Phi(z) d z \eta^{\prime}\left(\frac{c t}{2}\right)+\mathcal{O}\left(\varepsilon^{1 / 6}\right) .
\end{aligned}
$$

Finally, if we choose $\Phi$ so that

$$
\begin{aligned}
& \int_{-1}^{0} \Phi(z) d z=A_{1}, \quad \int_{0}^{1} \Phi(z) d z=A_{2}, \quad A_{1}+A_{2}=1 \\
& A_{1}\left(g\left(U_{L}\right)-2 c\right)+A_{2}\left(g\left(U_{R}\right)-2 c\right)=0,
\end{aligned}
$$

we see that (50) holds. This directly follows upon combining (60), (64), (67) and (68).

Theorem 6. For every fixed $t>0$, the function $v_{\varepsilon}$ given by (59) satisfies as $\varepsilon \rightarrow 0$

$$
v_{\varepsilon} \rightarrow v=\frac{t}{2} K \delta\left(x-\frac{c}{2} t\right)+\left\{\begin{array}{ll}
V_{L} & x<\frac{c}{2} t \\
V_{R} & x \geq \frac{c}{2} t
\end{array} \quad \text { in } \mathcal{D}^{\prime}(\mathbb{R}),\right.
$$

for $K$ defined in (52).

Proof: Take an arbitrary $\eta \in C_{0}^{1}(\mathbb{R})$. Using (57) we have:

$$
\begin{gathered}
\int v_{\varepsilon}(x, t) \eta(x) d x=\int_{-\infty}^{\varphi_{2}^{*}} V_{L} \eta(x) d x+\int_{\varphi_{1}^{*}}^{\infty} V_{R} \eta(x) d x+\int_{\varphi_{2}^{*}}^{\varphi_{1}^{*}} \frac{1}{\frac{\partial X}{\partial x_{0}}} \hat{v}\left(x_{0}\right) \eta(x) d x \\
+\int_{\varphi_{2}^{*}}^{\varphi_{1}^{*}}\left(\frac{1}{\frac{\partial X}{\partial x_{0}}} \int_{0}^{t} B_{1} K \frac{1}{\varepsilon^{1 / 3}} \Phi\left(\frac{X\left(x_{0}(x, t, \varepsilon), t\right)-\tilde{\phi}}{\varepsilon^{1 / 3}}\right) \frac{\partial X}{\partial x_{0}} d t^{\prime}\right) \eta(x) d x,
\end{gathered}
$$

where $x_{0}(x, t, \varepsilon)$ is the inverse function of the function $X=X\left(x_{0}, t, \varepsilon\right)$ defined in (57). Furthermore, here and in the sequel we imply $B_{1}=B_{1}\left(\rho\left(\tau\left(t^{\prime}\right)\right)\right)$.

Introducing the change of variables

$$
x=X\left(x_{0}, t, \varepsilon\right) \Rightarrow d x=\frac{\partial X}{\partial x_{0}} d x_{0},
$$


expression (70) becomes

$$
\begin{aligned}
& \int_{-\infty}^{\varphi_{2}^{*}} V_{L} \eta(x) d x+\int_{\varphi_{1}^{*}}^{\infty} V_{R} \eta(x) d x+\int_{-\varepsilon^{1 / 2}-\varepsilon^{1 / 3}}^{\varepsilon^{1 / 2}+\varepsilon^{1 / 3}} \hat{v}\left(x_{0}\right) \eta(X) d x_{0} \\
& +K \int_{-\varepsilon^{1 / 2}-\varepsilon^{1 / 3}}^{\varepsilon^{1 / 2}+\varepsilon^{1 / 3}} \int_{0}^{t} B_{1} \frac{1}{\varepsilon^{1 / 3}} \Phi\left(\frac{X\left(x_{0}, t, \varepsilon\right)-\tilde{\phi}}{\varepsilon^{1 / 3}}\right) \frac{\partial X}{\partial x_{0}} d t^{\prime} \eta(X) d x_{0} .
\end{aligned}
$$

We consider two last terms from (71).

Since $\hat{v}\left(x_{0}\right)$ is bounded, we have:

$$
\int_{-\varepsilon^{1 / 2}-\varepsilon^{1 / 3}}^{\varepsilon^{1 / 2}+\varepsilon^{1 / 3}} \hat{v}\left(x_{0}\right) \eta(X) d x_{0} \rightarrow 0 \quad \text { as } \quad \varepsilon \rightarrow 0 .
$$

Consider now the last term from (71):

$$
\begin{aligned}
& K \int_{-\varepsilon^{1 / 2}-\varepsilon^{1 / 3}}^{\varepsilon^{1 / 2}+\varepsilon^{1 / 3}} \int_{0}^{t} B_{1} \frac{1}{\varepsilon^{1 / 3}} \Phi\left(\frac{x_{0}-\tilde{\phi}}{\varepsilon^{1 / 3}}\right) \frac{\partial X}{\partial x_{0}} d t^{\prime} \eta(X) d x_{0} \\
& =K \int_{-\varepsilon^{1 / 2}-\varepsilon^{1 / 3}}^{X^{-1}\left(\varphi_{2}\right)} \int_{0}^{t} B_{1} \frac{1}{\varepsilon^{1 / 3}} \Phi\left(\frac{X\left(x_{0}, t, \varepsilon\right)-\tilde{\phi}}{\varepsilon^{1 / 3}}\right) \frac{\partial X}{\partial x_{0}} d t^{\prime} \eta(X) d x_{0} \\
& +K \int_{X^{-1}\left(\varphi_{2}\right)}^{X^{-1}\left(\varphi_{1}\right)} \int_{0}^{t} B_{1} \frac{1}{\varepsilon^{1 / 3}} \Phi\left(\frac{X\left(x_{0}, t, \varepsilon\right)-\tilde{\phi}}{\varepsilon^{1 / 3}}\right) \frac{\partial X}{\partial x_{0}} d t^{\prime} \eta(X) d x_{0} \\
& +K \int_{X^{-1}\left(\varphi_{2}\right)}^{\varepsilon^{1 / 2}+\varepsilon^{1 / 3}} \int_{0}^{t} B_{1}(\rho(\tau)) \frac{1}{\varepsilon^{1 / 3}} \Phi\left(\frac{X\left(x_{0}, t, \varepsilon\right)-\tilde{\phi}}{\varepsilon^{1 / 3}}\right) \frac{\partial X}{\partial x_{0}} d t^{\prime} \eta(X) d x_{0} .
\end{aligned}
$$

Then, repeating the procedure between formulas (61) and (67) we see that as $\varepsilon \rightarrow 0$ :

$$
\begin{aligned}
& K \int_{-\varepsilon^{1 / 2}-\varepsilon^{1 / 3}}^{X^{-1}\left(\varphi_{2}\right)} \int_{0}^{t} B_{1} \frac{1}{\varepsilon^{1 / 3}} \Phi\left(\frac{X\left(x_{0}, t, \varepsilon\right)-\tilde{\phi}}{\varepsilon^{1 / 3}}\right) \frac{\partial X}{\partial x_{0}} d t^{\prime} \eta(X) d x_{0} \rightarrow \frac{t}{2} K \eta\left(\frac{c}{2} t\right) \int_{0}^{1} \Phi(z) d z \\
& K \int_{X^{-1}\left(\varphi_{2}\right)}^{X^{-1}\left(\varphi_{1}\right)} \int_{0}^{t} B_{1} \frac{1}{\varepsilon^{1 / 3}} \Phi\left(\frac{X\left(x_{0}, t, \varepsilon\right)-\tilde{\phi}}{\varepsilon^{1 / 3}}\right) \frac{\partial X}{\partial x_{0}} d t^{\prime} \eta(X) d x_{0} \rightarrow 0 \\
& K \int_{X^{-1}\left(\varphi_{2}\right)}^{\varepsilon^{1 / 2}+\varepsilon^{1 / 3}} \int_{0}^{t} B_{1} \frac{1}{\varepsilon^{1 / 3}} \Phi\left(\frac{X\left(x_{0}, t, \varepsilon\right)-\tilde{\phi}}{\varepsilon^{1 / 3}}\right) \frac{\partial X}{\partial x_{0}} d t^{\prime} \eta(X) d x_{0} \rightarrow \frac{t}{2} K \eta\left(\frac{c}{2} t\right) \int_{-1}^{0} \Phi(z) d z
\end{aligned}
$$

where we used

$$
X\left(x_{0}, t, \varepsilon\right) \rightarrow \frac{c}{2} t \quad(\mathrm{cf} .(66))
$$

and

$$
B_{1}=B_{1}(\rho(\tau(t))) \rightarrow 1 / 2 \text { for every } t>0 \text { (cf. (23)). }
$$

Since $\int_{-1}^{0} \Phi(z) d z+\int_{0}^{1} \Phi(z) d z=1$, it is now clear that after letting $\varepsilon \rightarrow 0$ in $(71)$ we obtain (69).

5. ON A LINEARIZATION FOR AN ARBITRARY 2X2 HYPERBOLIC SYSTEM OF CONSERVATION LAWS

In this section we will give possible application of the method presented above on a Riemann problem for an arbitrary $2 \times 2$ system of conservation laws. So, consider 
the following system in one space dimension:

$$
\begin{aligned}
& \partial_{t} u+\partial_{x} f(u, v)=0, \\
& \partial_{t} v+\partial_{x} g(u, v)=0, \quad t \in \mathbb{R}^{+}, x \in \mathbb{R},
\end{aligned}
$$

where $f, g \in C^{1}\left(\mathbf{R}^{+} \times \mathbf{R}\right)$ with the following Riemann initial data:

$$
\begin{aligned}
& \left.u\right|_{t=0}=u_{0}(x)= \begin{cases}U_{L}, & x<0 \\
U_{R}, & x \geq 0\end{cases} \\
& \left.v\right|_{t=0}=v_{0}(x)= \begin{cases}V_{L}, & x<0 \\
V_{R}, & x \geq 0 .\end{cases}
\end{aligned}
$$

Passing to the Riemann invariants [4, Definition 7.3.1], as long as (74) admits classical solution, we can rewrite system (74) in the form:

$$
\begin{aligned}
& \partial_{t} \omega+\lambda_{1}(u, v) \partial_{x} \omega=0, \\
& \partial_{t} \eta+\lambda_{2}(u, v) \partial_{x} \eta=0,
\end{aligned}
$$

where $\lambda_{i}, i=1,2$, are eigenvalues of the Jacobian of flux matrix $\nabla(f(u, v), g(u, v))$, and $\omega=\omega(u, v)$ and $\eta=\eta(u, v),(u, v) \in \mathbf{R}^{2}$, are the Riemann invariants.

It is clear that it is much easier to work with the former diagonal system then with original system (74). But, with initial data (75), system (74) in general does not admit the classical solution. Therefore, in order to connect properly (74) and (76), instead of initial data $\omega\left(u_{0}, v_{0}\right)$ and $\eta\left(u_{0}, v_{0}\right)$, we augment (74) with the following smooth perturbations of $\omega\left(u_{0}, v_{0}\right)$ and $\eta\left(u_{0}, v_{0}\right)$, respectively:

$$
\begin{aligned}
& \left.\omega\right|_{t=0}=\omega_{0 \varepsilon}(x)= \begin{cases}\omega\left(U_{L}, V_{L}\right), & x>-A(\varepsilon) \\
\omega\left(u_{0 \varepsilon}^{1}(x), v_{0 \varepsilon}^{1}(x)\right), & -A(\varepsilon) \leq x<A(\varepsilon), \\
\omega\left(U_{R}, V_{R}\right), & x \geq A(\varepsilon),\end{cases} \\
& \left.\eta\right|_{t=0}=\eta_{0 \varepsilon}(x)= \begin{cases}\eta\left(U_{L}, V_{L}\right) & x<-B(\varepsilon) \\
\eta\left(u_{0 \varepsilon}^{2}(x), v_{0 \varepsilon}^{2}(x)\right), & -B(\varepsilon) \leq x<B(\varepsilon) \\
\eta\left(U_{R}, V_{R}\right), & x \geq B(\varepsilon),\end{cases}
\end{aligned}
$$

where $A$ and $B$ are appropriate positive functions tending to zero as $\varepsilon \rightarrow 0$, and $u_{0 \varepsilon}^{i}$ and $v_{0 \varepsilon}^{i}, i=1,2$, are such that $\left.\omega\right|_{t=0}$ and $\left.\eta\right|_{t=0}$ are Lipschitz continuous functions.

According to the standard theory of scalar conservation laws, Cauchy problem (76), (77) will have classical solution till the moment $\min \left\{t^{*}, t^{* *}\right\}$, where

$$
\begin{aligned}
t^{*} & =\frac{1}{\max _{x \in(-A(\varepsilon), A(\varepsilon))} \partial_{x} \lambda_{1}\left(u_{0 \varepsilon}^{1}(x), v_{0 \varepsilon}^{1}(x)\right)}, \\
t^{* *} & =\frac{1}{\max _{x \in(-B(\varepsilon), B(\varepsilon))} \partial_{x} \lambda_{2}\left(u_{0 \varepsilon}^{2}(x), v_{0 \varepsilon}^{2}(x)\right)} .
\end{aligned}
$$

As before, the idea is to modify characteristics for a small parameter $\varepsilon$ so that its intersection is avoided, and then to continue classical solution along such 'new characteristics'. Letting $\varepsilon \rightarrow 0$ we should recover singularity which solves our original Riemann problem.

So, instead of system (76), (77) we should solve

$$
\begin{aligned}
& \partial_{t} \omega_{\varepsilon}+\lambda_{1}(u, v) \partial_{x}\left(\left(B_{2}^{\omega}-B_{1}^{\omega}\right) \omega_{\varepsilon}+c_{1}(t, x) B_{1}^{\omega} \omega_{\varepsilon}\right)=F(t, x, \varepsilon), \\
& \partial_{t} \eta_{\varepsilon}+\lambda_{2}(u, v) \partial_{x}\left(\left(B_{2}^{\eta}-B_{1}^{\eta}\right) \eta_{\varepsilon}+c_{2}(t, x) B_{1}^{\eta} \eta_{\varepsilon}\right)=G(t, x, \varepsilon),
\end{aligned}
$$


with initial data (77) for appropriately chosen $u_{0 \varepsilon}^{i}$ and $v_{0 \varepsilon}^{i}, i=1,2$ (see (80)).

Furthermore,

$$
B_{i}^{\omega}=B_{i}\left(\rho_{\omega}\right), \quad B_{i}^{\eta}=B_{i}\left(\rho_{\eta}\right), \quad i=1,2,
$$

where the functions $B_{i}, i=1,2$, are defined in Theorem 3 , and

$$
\rho_{\omega}=\frac{\varphi_{2}^{\omega}(t, \varepsilon)-\varphi_{1}^{\omega}(t, \varepsilon)}{\varepsilon}, \quad \rho_{\eta}=\frac{\varphi_{2}^{\eta}(t, \varepsilon)-\varphi_{1}^{\eta}(t, \varepsilon)}{\varepsilon},
$$

where $\varphi_{1}^{\omega}$ and $\varphi_{1}^{\eta}$ are characteristics corresponding to $\omega_{\varepsilon}$ and $\eta_{\varepsilon}$ from (79), and emanating from $A(\varepsilon)$ and $B(\varepsilon)$, respectively. Similarly, $\varphi_{1}^{\omega}$ and $\varphi_{1}^{\eta}$ are characteristics corresponding to $\omega_{\varepsilon}$ and $\eta_{\varepsilon}$ from (79), and emanating from $-A(\varepsilon)$ and $-B(\varepsilon)$, respectively.

Remark 7. Notice that we can take $\rho_{\eta}$ instead of $\rho_{\omega}$ or vice versa since we expect to have blow up of both Riemann invariants at the same time. So, depending on the case that we are considering, we will choose one of the functions $\rho_{\eta}$ or $\rho_{\omega}$.

Other functions appearing in (79) need to be determined so that the family of solutions to (79), (77) represents the weak asymptotic solution to system (74), (75).

What we hope is that the situation will be the same as in the case of system (3), (2) at least in the case of special values for $U_{L}, U_{R}, V_{L}$ and $V_{R}$. Namely, after the intersection of characteristics, the function $B_{2}-B_{1}$ should eliminate nonlinearity with expense to a 'bad' righthand side. Thus, instead of nonlinear conservation law we would obtain two transport equations which are in general easier to solve [1].

As we can see, everything depends on the function $B_{i}, i=1,2$, and these functions depend only on extremal characteristics (i.e. those emanating from $\pm A(\varepsilon)$ and $\pm B(\varepsilon)$ ). Therefore, if we want to have smooth solution all the way till the moment of intersection of extremal characteristics, we must determine the functions $u_{0}$ and $v_{0}$ from (77) so that all the characteristics corresponding to $\omega_{\varepsilon}$ and $\eta_{\varepsilon}$, and emanating from the intervals $(-A(\varepsilon), A(\varepsilon))$ and $(-B(\varepsilon), B(\varepsilon))$, respectively, intersects in the same point.

From (78) we have effective way for determining the functions $u_{0 \varepsilon}^{i}$ and $v_{0 \varepsilon}^{i}$, $i=1,2$. It is clear that it has to be:

$$
\begin{aligned}
& \partial_{x} \lambda_{1}\left(u_{0 \varepsilon}^{1}(x), v_{0 \varepsilon}^{1}(x)\right)=K, \quad x \in(-A(\varepsilon), A(\varepsilon)), \\
& \partial_{x} \lambda_{2}\left(u_{0 \varepsilon}^{2}(x), v_{0 \varepsilon}^{2}(x)\right)=K, \quad x \in(-B(\varepsilon), B(\varepsilon)) .
\end{aligned}
$$

for a positive constant $K$.

Realization of the procedure described in the current section will be the subject of further investigation. We believe that it could give new results in the case of hyperbolic systems whose Riemann problems do not admit solutions consisting from admissible elementary wave combinations, i.e. combinations of Lax admissible shock and rarefaction waves (see e.g. one dimensional Brio system [16]) .

\section{BIBLIOGRAPHY}

[1] L. Ambrosio, Transport equation and Cauchy problem for BV vector fields, Inventiones Mathematicae, 158 (2004), 227-260.

[2] F. Bachmann, J. Vovelle, Existence and uniqueness of entropy solution of scalar conservation law with a flux function involving discontinuous coefficients, Comm. Partial Differential Equations, 31: 371-395 (2006)

[3] G-Q. Chen, H. Liu, Formation of $\delta$-shocks and vacuum states in the vanishing pressure limit of solutions to the Euler equations for isentropic fluids, SIAM J. Math. Anal. 34 (2003), no. 4, 925-938. 
[4] C. M. Dafermos Hyperbolic Conservation Laws in Continuum Physics, Berlin; Heidelberg; New York; Barcelona; Hong Kong; London; Milan; Paris; Singapore; Tokyo: Springer, 2000.

[5] V. G. Danilov, Weak asymptotic solution of phase-field system in the case of confluence of free boundaries in the Stefan problem with underheating European Journal of Applied Mathematics, 2007; 18 (5), 537-570

[6] V. G. Danilov, Generalized Solution Describing Singularity Interaction, International Journal of Mathematics and Mathematical Sciences, Volume 29, No. 22. February 2002, pp. 481-494.

[7] V. G. Danilov, D. Mitrovic, Delta shock wave formation in the case of triangular hyperbolic system of conservation laws, to appear in J. of Differential Equations, available at http://www.math.ntnu.no /conservation/2006/057.html

[8] V. G. Danilov, D. Mitrovic, Weak asymptotic of shock wave formation process, Journal of Nonlinear Analysis - Theory, Methods and Applications, 61(2005) 613-635.

[9] V. G. Danilov, G. A. Omelianov, V. M. Shelkovich, Weak Asymptotic Method and Interaction of Nonlinear Waves in: M.Karasev (Ed.), Asymptotic Methods for Wave and Quantum Problems, American Mathematical Society Translation Series, vol. 208, 2003, pp. 33-165.

[10] V.G. Danilov, V.M.Shelkovich, Dynamics of propagation and interaction of $\delta$-shock waves in conservation law system, J. Differential Equations 211 (2005) 333-381.

[11] V.G. Danilov, V.M.Shelkovich, Delta-shock wave type solution of hyperbolic systems of conservation laws, Quart. Appl. Math. 63 (2005), 401-427

[12] V. G. Danilov, V. M. Shelkovich, Propagation and Interaction of shock waves of quasilinear equations, Nonlinear Stud. 8 (1) (2001) 135-169.

[13] X. Ding, Z. Wang, Existence and Uniqueness of Discontinuous Solution defined by LebesgueStieltjes integral, Sci. China Ser. A, 39 (1996), no.8., 807-819

[14] G. Ercole, Delta-shock waves as self-similar viscosity limits, Quart. Appl. Math. LVIII (1) (2000) 177-199.

[15] D. Gilbarg, N. Trudinger, Elliptic Partial Differential Equations of Second Order, SpringerVerlag-Berlin Heidelberg New York Tokyo 1983

[16] B. Hayes, P. G. LeFloch, Measure-solutions to a strictly hyperbolic system of conservation laws, Nonlinearity 9 (1996), 1547-1563.

[17] F. Huang, Existence and Uniqueness of Discontinuous Solutions for a Class of Non-strictly Hyperbolic System, Advances in nonlinear partial differential equations and related areas (Beijing, 1997), 187-208, World Sci. Publ., River Edge, NJ, 1998.

[18] F. Huang, Existence and uniqueness of discontinuous solutions for a hyperbolic system Proc.Roy.Soc.Edinburgh Sect. A 127 (1997), no. 6, 1193-1205.

[19] F. Huang, Weak solution to pressureless type system, Comm. Partial Differential Equations 30 (2005), no. 1-3, 283-304.

[20] S. Hwang, Nonlin. diffusive-dispersive limit for scalar multidim. cons. laws, J. Differential Equations, 225 (2006) 90-102.

[21] K. T. Joseph, A Riemann problem whose viscosity solution contains $\delta$ measures, Asymptotic Analysis 7 (1993) 105-120.

[22] B. L. Keyfitz, H. C. Krantzer, Spaces of weighted measures for conservation laws with singular shock solutions, J. Differential Equations 118 (1995) 420-451.

[23] S. N. Kruzhkov, First order quasilinear quations in several independent variables, Math. USSR Sb. 10, (1970), 217-243.

[24] P. G. LeFloch, An existence and uniqueness result for two nonstrictly hyperbolic systems, IMA Volumes in Math. and its Appl., "Nonlinear evolution equations that change type", ed. B.L. Keyfitz and M. Shearer, Springer Verlag, Vol. 27, 1990, pp. 126-138.

[25] P. L. Lions, B. Perthame, E. Tadmor, A kinetic formulation of multidimensional scalar conservation law and related equations, J. Amer. Math. Soc., Vol 7, 1994, pp. 169-191.

[26] Y.-G. Lu, Existence of Global Entropy Solutions of a Nonstrictly Hyperbolic System, Arch. Rat. Mach. Anal. 178 (2005) 287-299

[27] D. Mitrovic, M. Nedeljkov, Delta shock waves as a limit of shock waves, J. of Hyperbolic Differential Equations, Vol. 4, No. 4 (2007), 1-25.

[28] D. Mitrovic, J. Susic, Global in time solution to Hopf equation and application to a nonstrictly hyperbolic system of conservation laws, Electronic J. of Differential Equations, Vol. 2007(2007), No. 114, pp. 1-22.

[29] F. Murat, Compacite par compensation, Ann. Scuola Norm. Sup. Pisa 5, 489-507 (1978)

[30] M. Nedeljkov, Singular shock waves in interactions, to appear in Q. Appl. Math. 
[31] M. Nedeljkov, Delta and singular delta locus for one-dimensional systems of conservation laws, Math. Meth. Appl. Sci. 27 (2004), 931-955.

[32] E. Yu. Panov, V. M. Shelkovich, $\delta^{\prime}$-shock waves as a new type of solutions to systems of conservation laws, J.Differential Equations 228 (2006), no. 1, 49-86.

[33] B. Perthame, Kinetic approach to systems of conservation laws Journees equations aux derivees partielles (1992), Art. No. 8, 13 p.

[34] B.Perthame, Uniqueness and error estimates in first order quasilinear conservation laws via the kinetic entropy defect measure, J.Math.Pures Appl., 77 (1998), 1055-1064.

[35] S. A. Sazhenkov, The genuinely nonlinear Graetz-Nusselt ultraparabolic equation, Siberian Mathematical Journal, Vol. 47, No. 2, pp. 355-375, 2006.

[36] W. Sheng, T. Zhang, The Riemann problem for transportation equations in gas dynamics, Mem. Amer. Math. Soc. 137 (645) (1999) 1-77.

[37] D. Tan, T. Zhang, Y. Zheng, Delta shock waves as a limits of vanishing viscosity for a system of conservation laws, J. Differential Equations 112 (1994) 1-32.

[38] L. Tartar, Compensated compactness and application to partial differential equations, Nonlin. Anal.and Mech.: Heriot-Watt symposium, Vol. IV, Res. Notes Math., 39 (1979) 136-212.

[39] H. Yang, Riemann problems for class of coupled hyperbolic system of conservation laws, Journal of Differential Equations, 159(1999) 447-484.

Velibor Bojkovic, University of Montenegro, Faculty of Mathematics, Cetinjski PUt BB, 81000 Podgorica, Montenegro

E-mail address: vlavelbo@cg.yu

Vladimir G. Danilov, Moscow Technical University of Communication and Informatics, Aviamotornaya 8A, 111024 Moscow, Russia

E-mail address: danilov@miem.edu.ru

Darko Mitrovic, University of Montenegro, Faculty of Mathematics, Cetinjski put BB, 81000 Podgorica, Montenegro

E-mail address: darko@rc.pmf.cg.ac.yu 